\title{
OntoFilm: A Core Ontology for Film Production
}

\author{
Ajay Chakravarthy, Richard Beales, Nikos Matskanis, and Xiaoyu Yang \\ IT Innovation Centre, 2 Venture Road, \\ Southampton, SO16 7NP United Kingdom \\ \{ajc, rmb, nm, kxy\}@it-innovation.soton.ac.uk
}

\begin{abstract}
In this paper we present OntoFilm, a core ontology for film production. OntoFilm provides a standardized model which conceptualizes the domain and workflows used at various stages of the film production process starting from pre-production and planning, shooting on set, right through to editing and post-production. The main contributions in this paper are: we discuss how OntoFilm models the semantics necessary to interpret these workflows consistently for all users (Directors, DoP's, grips, post-production, lighting). We also discuss how our ontology forms a common bridge between the low level descriptive metadata generated for the video footage and the high level semantics used in software tools during the production process.
\end{abstract}

Keywords: Ontologies, Semantic Web, Film Production, Post Production, Digital Media, Semantic Gap.

\section{Introduction}

The process of film production is lengthy, time consuming and often an expensive affair. Film directors currently rely on paper based storyboards or manually-created 'pre-vis' animations to record and express their creative intent on how a scene could be shot. There is no formal way to describe this intent. Similarly in post production a tremendous amount of metadata is available describing the footage that has been captured, but this is in the form of paper notes and must be sorted and input into the postproduction software manually. This is a time-consuming, laborious and error-prone process. There is a need for a standardized knowledge model which will act as a common knowledge sharing platform between the software tools used throughout film production. This research is being carried out as part of the ANSWER $^{1}$ project. ANSWER is a novel approach to the creative process of film making. ANSWER introduces a symbolic language for directors to create detailed scene descriptions, much like a musician would use music scores to compose a song. ANSWER also aims to provide tools and technologies which will enable scene authoring through filming notation. Users (directors) will be able to define and visualize film scenes before shooting begins. The aim of our research within ANSWER is to create a conceptual knowledge model which facilitates knowledge flow among the various software components used and is a first step towards bridging the semantic gap between high level pre-production

${ }^{1}$ www.answer-project.org/ 
concepts and low level post-production metadata. In its initial stages OntoFilm is mainly concerned with modelling film production. However, the film and games industries are converging in the way that their creative content is authored, so we will extend the results to include the needs of the games industry, thus offering a bridge between digital media production and animation for game design. In this paper we will present how we have attempted to model the workflow in three main stages of film production, i.e. pre-production, on-location, and post-production. Prior to starting development on OntoFilm we have studied state of the art multimedia ontologies such as MPEG-7 [2] and COMM [1]. We have reused concepts from the MPEG-7 ontology (e.g. for Camera Motion, Motion Trajectories and Motion Activity).

\section{Pre-production Model}

The pre-production model covers the concepts used during the planning stage of the film. Script writers and film directors use paper based scripts and story boards to plan shots within the film. Often directors add additional notes on script elements to record their creative ideas. This additional metadata is very useful in post-production when locating footage and during video and dialogue synchronization. However paper based scripts cannot be used directly to produce machine understandable metadata. OntoFilm introduces the concept of Shooting Script element. After consultation with directors and analyzing existing film and television scripts we have identified the main elements of a movie script and their relationships such as Scene, Shot, Character Name, Dialogue, Parenthetical and Transition Element. The Movie Script Markup Language (MSML) is a movie script schema specification developed as part of the ANSWER ontology specification after studying existing schemas used within the film domain such as Celtx ${ }^{2}$. MSML is encoded within OntoFilm and provides the semantics necessary for software tools to annotate and parse movie scripts according to this schema. Figure 1 shows the conceptual schema of MSML.

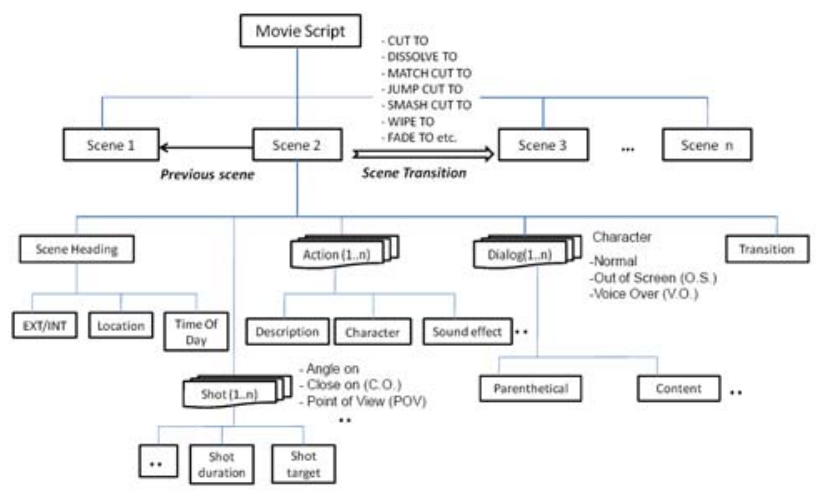

Fig. 1. Movie Script Markup Language conceptual schema

\footnotetext{
${ }^{2}$ http://wiki.celtx.com/index.php?title=The_Script_Editor
} 
The graphical user interface developed within ANSWER allows directors to visualize and annotate scripts just as they would on a paper based script, the difference being all the concepts being annotated are persisted as OWL instances. Further the director can select any element on the script and add free text annotations to record his/her creative intent. Other pre-production concepts include various physical setups required on set such as Optical Attachments, Optical Components, Costume, Props, Shots (e.g. long shot, POV shot, medium shot etc). Detailed descriptions of the aforementioned classes are published in [4]. We have done initial experimentation with $\mathrm{SWRL}^{3}$ based rule modelling e.g. for automatic classification of the different Cameras used during filming. Figure 2 shows a rule for classifying Film Camera.

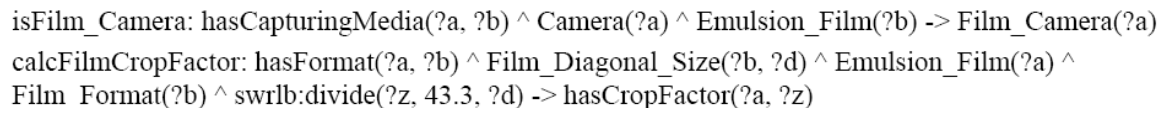

Fig. 2. SWRL rule showing classification conditions for Film Camera

The isFilm_Camera rule classifies the Camera instance according to if it has Emulsion Film as capturing media. The camera's hasCropFactor data property can then be calculated by taking into account its classification, film or sensor size by the calcFilmCropFactor and calcDigitalCropFactor.

\section{On-Set Production Model}

Directors often follow a set of filming conventions while shooting a scene. Although these are not mandatory, these rules are recommended to avoid causing confusion to the viewing audience. In OntoFilm we have conceptualized these rules with Film Grammar concepts. A few examples of these rules include:

- 180 Degree Rule: This rule deals with the spatial relation between characters on-screen. It is used to maintain consistent screen direction between characters or between a character and an object.

- 30 Degree Rule: If a shot is going from one character to another without an intervening shot of something else, the camera angle should change by at least 30 degrees.

The on set production model also includes the Director Notation (DN) ontology. DN is a symbolic language which can be used to describe detailed scene descriptions during film shooting [3]. The graphical user interface developed in ANSWER will allow directors to create detailed scene descriptions using DN. A rule engine automatically translates these DN scores and generates animates pre-visualizations. The DN ontology is a system level ontology which acts as a bridge between the user enabled tools for using DN to describe a scene and the rule engine which translates the DN scores into animations. Details of the DirectorNotation ontology will not be expanded since it's outside the scope of this paper.

\footnotetext{
${ }^{3}$ http://www.w3.org/Submission/SWRL/
} 


\section{Post-Production Model}

Our approach to modelling Post-Production for OntoFilm has been to focus on the workflow involved, as it was considered this would draw attention to the points at which metadata was lost or needed to be transferred manually. Significant amount of machine-readable metadata is automatically generated in post production to describe the footage from the point at which it is ingested (digitised), e.g. descriptions of media on which the files were held and software processes applied to alter the visual appearance of the footage. Furthermore, low-level video features, such as regions of colour or movement, can both be annotated automatically using the MPEG-7 standard. However there is a stark disconnect between these various technical parameters and the high-level features that they contain or represent. The result is that post-production operators can easily identify footage that had been scanned by a particular machine, but not find a sequence of shots that contained a scene description e.g. protagonists in a car chase - this information is buried in the reams of paper notes that accompany the production ${ }^{4}$. We have modelled the various post-production steps, e.g. Edit_Room (which involves assembly of selected material from video footage produced on a day to day basis), Commercial_Preview (where a candidate edit is screened in a cinema to judge audience reaction), and linked them not only technical descriptions of the media involved, but also to scene descriptions from the On-Set Production model and planning notes from the Pre-Production model. In this way, it is now possible to construct a unified semantic representation of the action portrayed on scene, the actors, props and locations involved, the planning rationale for creating the scene and the technical means with which it was acquired and the processing applied in post. For example the low level footage descriptor Scene Tint (which is the predominant colour of the shot) relates to the high level concept of Content (which describes the mood the scene).

\section{Conclusions and Future Work}

The OntoFilm ontology is being developed after direct consultations with film directors $\left(\mathrm{STEFI}^{5}\right.$ Productions), post production software developers (DFT ${ }^{6}$ ) and game developers (Larian ${ }^{7}$ Studios). The feedback from these collaborative meetings confirmed the need for development of a common ontological framework for the digital media domain, which will act as a bridge for knowledge transfer between these domains consistently for all users. We are currently investigating integrating OntoFilm with the COMM API. The COMM ontology combines the advantages of extensibility and scalability of a web-based solution with the accumulated experience of MPEG-7 [1]. The main advantage of doing this would be software programs will be easily able to access the ontology through programmatic interfaces. We are also looking at

\footnotetext{
${ }^{4}$ This information has been gathered after having technical meetings with post production teamDFT Bones@Weiterstadt, Germany.

${ }^{5} \mathrm{http}: / /$ www.stefi.gr/

${ }^{6} \mathrm{http}: / / \mathrm{www} . \mathrm{dft}-\mathrm{film} . \mathrm{com} /$

${ }^{7}$ http://www.larian.com/
} 
current efforts in the field of digital media ontologies, namely the $\mathrm{iMP}^{8}$ and Salero 9 projects. However our work differs from these multimedia ontologies because OntoFilm is specific to film production and covers this domain in great depth with a rich set of semantics. Software programs developed for digital media production will be able to leverage our ontology directly.

\section{References}

1. Arndt, R., Troncy, R., Staab, S., Hardman, L., Vacura, M.: COMM: Designing a WellFounded Multimedia Ontology for the Web. In: Aberer, K., Choi, K.-S., Noy, N., Allemang, D., Lee, K.-I., Nixon, L.J.B., Golbeck, J., Mika, P., Maynard, D., Mizoguchi, R., Schreiber, G., Cudré-Mauroux, P. (eds.) ASWC 2007 and ISWC 2007. LNCS, vol. 4825, pp. 30-43. Springer, Heidelberg (2007)

2. Martinez, M.J.: MPEG-7 Overview: International organization for standardisation (ISO//IEC/JTCI/SC29/WG11). Coding of moving pictures and audio. Palma be Mallorca (October 2004)

3. Yannopoulos, A., Savrami, K., Varvarigou, T.: DirectorNotation as a Tool for AmI \& Intelligent Content: an Introduction by Example. Accepted for publication in the International Journal of Cognitive Informatics and Natural Intelligence, IJCiNi (2008)

4. D4.1B: Mid-Term Ontology Report, ANSWER Project Deliverable (May 2009)

\footnotetext{
${ }^{8} \mathrm{http}: / /$ imp-project.eu/

${ }^{9} \mathrm{http}: / /$ www.salero.eu/
} 